\title{
Política de formación académica con enfoque de RSU para la Universidad de Panamá, planificación para la acción
}

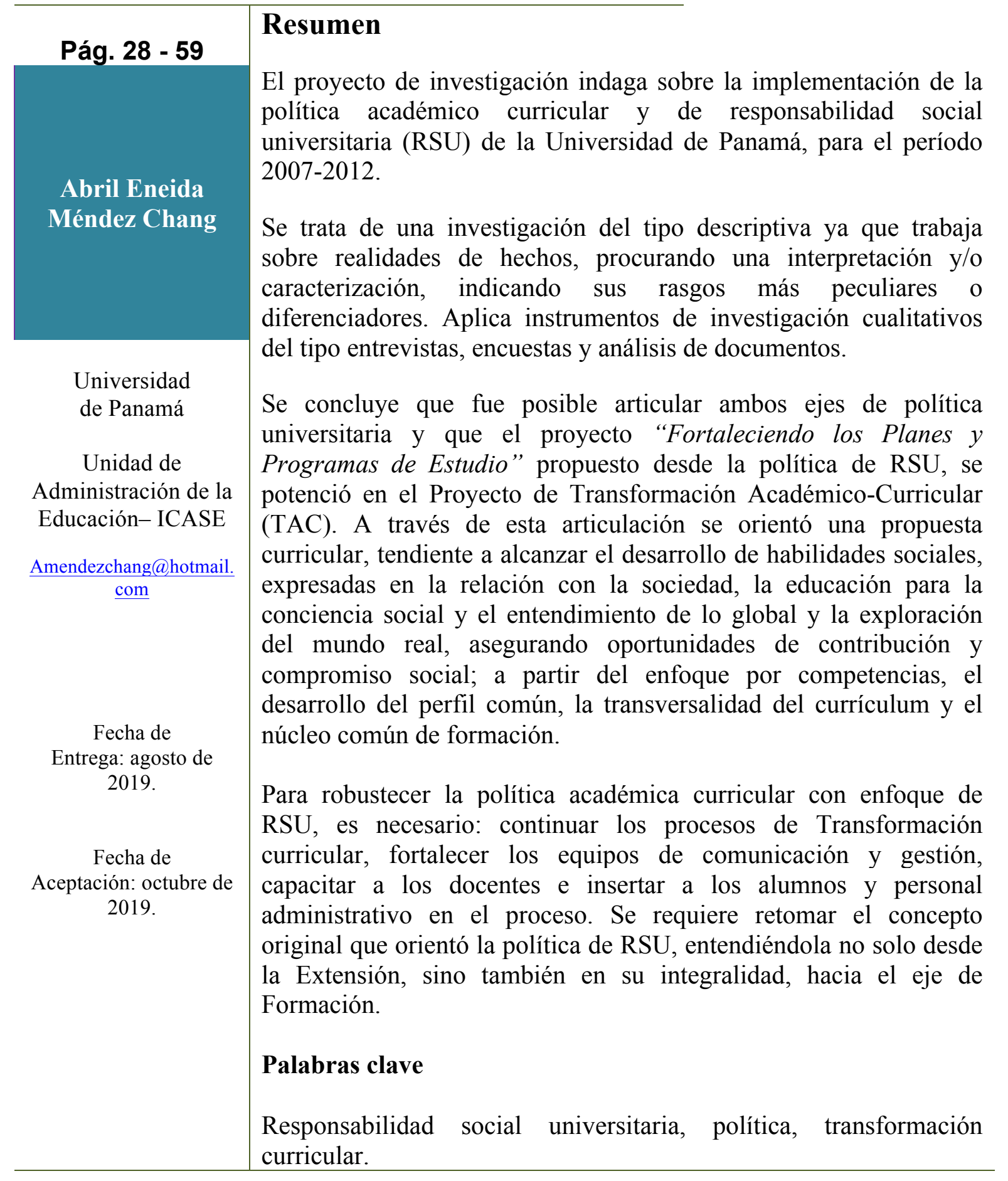




\title{
An academic training policy with an USR approach for the Universidad de Panama, planning for action
}

\begin{abstract}
This research project studies the implementation of the academic curriculum and university social responsibility (RSU) policies of the Universidad de Panama, for years 2007-2012.

It is a descriptive research for it works on real facts, seeking an interpretation and / or characterization highlighting its most peculiar or differentiating features. There is a priority for the application of qualitative research instruments such as interviews, surveys and document analysis.
\end{abstract}

The conclusion is that it was possible to articulate both university policy axes and that the project "Strengthening the Study Plans and Curriculum" proposed from the USR policy was enhanced by the Academic-Curricular Transformation Project (ACT). Thanks to this articulation a curricular proposal was oriented, aimed at achieving the development of social skills, expressed in the relationship with society, education for social conscience and understanding of the global and real world exploration, which led to ensure opportunities for social contribution and commitment; based on the competency approach, the development of common profile, the mainstreaming of the curriculum and the common core of training.

\section{Keywords}

University social responsibility, policy, curricular transformation.

REVISTA ANUAL ACCIÓN Y REFLEXIÓN EDUCATIVA, N 45

enero, 2020

SSN L 2664-3775 
Política de formación académica con enfoque de RSU para la

Universidad de Panamá, planificación para la acción.

\section{INTRODUCCIÓN}

La Conferencia de Educación Superior de 2009 reconoce que la educación superior tiene la responsabilidad social de hacer avanzar nuestra comprensión de problemas polifacéticos con dimensiones sociales, económicas, científicas y culturales, así como nuestra capacidad de hacerles frente. Por ello establece que la educación superior debe asumir el liderazgo social en materia de creación de conocimientos de alcance mundial para abordar retos mundiales, entre los que figuran la seguridad alimentaria, el cambio climático, la gestión del agua, el diálogo intercultural, las energías renovables y la salud pública. De allí la importancia de la Responsabilidad Social Universitaria como eje de política universitaria, entendida como un desafío, que supone el repensar del modelo universitario en todas sus funciones, y en especial, en lo relativo a la formación de profesionales íntegros y comprometidos con las soluciones de los problemas del desarrollo.

La Universidad de Panamá, comprometida con el desarrollo nacional, a través de su permanente vinculación con los sectores más excluidos de la sociedad y atendiendo a su mandato legal (Ley 24 Orgánica de la Universidad de Panamá de 14 de julio de 2005); orienta su planificación estratégica, hacia el logro de políticas, entre las que destacan para el período 2007-2011: La de Responsabilidad Social Universitaria y la de Formación Académica-Curricular.

1. Proyección universitaria,

2. Formación académica y transformación curricular,

3. Producción y desarrollo del conocimiento por la investigación,

4. Alianzas estratégicas con el sistema educativo,

5. Mejoramiento del recurso humano,

6. Innovación tecnológica,

7. Ambiente y gestión de riesgo y,

8. Responsabilidad Social Universitaria (RSU).

REVISTA ANUAL ACCIÓN Y REFLEXIÓN EDUCATIVA, N 45

enero, 2020

ISSN L 2664-3775 
La Política Académica-Curricular (2007-2011), insertó el Eje estratégico 2 de Formación Académica y Transformación Curricular el cual consideró el Proyecto de Transformación Académica-Curricular. Este proyecto, aprobado por el Consejo Académico de la Universidad de Panamá (Sesión $N^{\circ} 44$ del 27 de octubre de 2004), procura orientar y asegurar la congruencia de la oferta educativa, con las necesidades sociales, culturales y académicas del país y las tendencias del desarrollo científico tecnológico.

Por otro lado, la política Responsabilidad Social Universitaria desarrolló, para el Eje Estratégico 6, además de la creación de núcleos gestores e integrar espacios para la RSU; un proyecto dirigido a "Fortalecer los Planes y Programas de estudio desde el enfoque de la Responsabilidad Social Universitaria”.

De lo anterior resulta interesante verificar lo avanzado por el proyecto de Transformación Académico-Curricular de la Universidad de Panamá, a la luz de la teoría de la RSU; para de esta manera justificar la viabilidad de una nueva propuesta de política de RSU que potencie el eje de Formación Académica.

\section{PRECEPTOS TEÓRICOS SOBRE LA RESPONSABILIDAD SOCIAL UNIVERSITARIA PARA EL EJE DE FORMACIÓN}

Los preceptos teóricos sobre los que se sujeta la RSU, están fundamentados por sus concepciones descriptivas con relación a los compromisos, demandas y prospecciones sobre el desarrollo y las implicancias de las universidades panameñas con la sociedad y sus entornos naturales, comunitarios, académicos-sociales-humanísticos y tecnológicos. la Universidad de Panamá ha suscrito el compromiso social para producir un cambio relacionado con el bienestar social y cultural a partir de un proceso de transformación curricular, que permita enfrentar los desafíos relacionados a la planificación curricular; para lograr aprendizajes significativos que aseguren la formación integral del egresado.

REVISTA ANUAL ACCIÓN Y REFLEXIÓN EDUCATIVA, N 45

enero, 2020

SSN L 2664-3775 
Política de formación académica con enfoque de RSU para la

Universidad de Panamá, planificación para la acción.

\section{UNIVERSIDAD Y SU COMPROMISO SOCIAL PARA EL CAMBIO}

El rol de liderazgo de la universidad como ente de cambio social es resaltado durante la Conferencia Mundial sobre la Educación Superior - 2009 denominada "La nueva dinámica de la educación superior y la investigación para el cambio social y el desarrollo", en la cual se señala que la educación superior deberá crear conocimientos de alcance mundial para abordar los retos mundiales que aseguren la sostenibilidad (la seguridad alimentaria, el cambio climático, la gestión del agua, el diálogo intercultural, las energías renovables y la salud pública, entre otros); y contribuir con ello a la formación de ciudadanos responsables. Se insta a los centros de educación superior, a que en el desempeño de sus funciones primordiales (investigación, enseñanza y servicio a la comunidad) y en un contexto de autonomía institucional y libertad académica, promuevan la interdisciplinaridad, el pensamiento crítico y la ciudadanía activa. Para ello se advierte, que además de poner atención a los aspectos de calidad, pertinencia, eficacia y transparencia; está la responsabilidad social, aspecto esencial para "contribuir a la formación de ciudadanos dotados de principios éticos, comprometidos con la construcción de la paz, la defensa de los derechos humanos y los valores de la democracia" (UNESCO, 2009).

La Declaración final de la Conferencia destaca aspectos tales como:

- El rol ético de las universidades "convertirse en centros que anticipen, adviertan y prevean problemas futuros" (artículo 2).

- La necesidad de "creación de conocimiento". La universidad "debe acrecentar el conocimiento mediante la investigación en las ciencias, las artes y las humanidades, y la diseminación de sus resultados" (artículo 5).

- Que la educación superior debe reforzar su servicio a la sociedad y en especial sus actividades para eliminar la pobreza, la intolerancia, utilizando principalmente

REVISTA ANUAL ACCIÓN Y REFLEXIÓN EDUCATIVA, N 45

enero, 2020

ISSN L 2664-3775 
enfoques transdisciplinarios e interdisciplinarios en el análisis de los temas y problemas. (artículo 6).

- La universidad debe "reforzar la cooperación con el mundo del trabajo y el análisis y previsión de las necesidades de la sociedad” (artículo 7).

- La universidad debe "situar a los estudiantes y sus necesidades en el centro de sus preocupaciones y considerarlos responsables del proceso de renovación de la enseñanza superior" (artículo 10).

Se aspira a que la universidad forme ciudadanos responsables, atentos a los problemas que le rodean, con plena disposición para participar y liderizar procesos; una universidad donde se forme una conciencia crítica respecto a los problemas sociales que le afectan.

Una universidad que se considera como socialmente responsable debería visualizarse como: respetuosa con el ambiente, segura y saludable para sus empleados (docentes y administrativos) y alumnos, con igualdad de oportunidades y garante de la diversidad cultural, comprometida con las causas sociales y con la cooperación para el desarrollo, justa y transparente, que participa en proyectos de innovación e investigación pertinentes y que responden a las demandas de la sociedad, que genera conocimiento en sostenibilidad y educación ambiental, que es motor en la sociedad y que interactúa con la comunidad dando respuestas a sus problemas.

Entendido así, todas las funciones de la organización universitaria: docencia, investigación, servicios y extensión deberán estar orientadas a atender las demandas sociales. La RSU considera el desarrollo de prácticas educativas que propicien el compromiso social, lleven a la reflexión de los problemas y la resolución de estos.

En el caso de América Latina, la Responsabilidad Social Universitaria, se gesta formalmente en la Declaración de Bello Horizonte (UNESCO-IESALC, 2007), en el cual se indica que la 
Política de formación académica con enfoque de RSU para la

Universidad de Panamá, planificación para la acción.

universidad debe ser capaz de atender los desafíos relacionados con la erradicación de la pobreza, la intolerancia, la violencia, el analfabetismo, el hambre, el deterioro del medio

ambiente y las enfermedades, es decir, deben sumarse y colaborar con las acciones tendientes al logro de los Objetivos del Milenio y de la Educación para Todos.

Se destaca, así mismo, el "Compromiso Social de las Universidades de América Latina y El Caribe", la necesidad de generar cambios estratégicos, tales como:

- "Del voluntariado y la filantropía, al compromiso ético con la justicia social y el ejercicio de derechos.

- De la acción coyuntural, dispersa y episódica, a las líneas programáticas de largo alcance.

- De la realización de actividades de compromiso social por áreas de bajo nivel jerárquico de las instituciones, a su incorporación en las propias misiones institucionales.

- De la acción aislada y sectorial, a las sinergias en función de proyectos de país.

- De la extensión como servicio de transferencia, a los encuentros sociales, el diálogo de saberes, la construcción de conocimiento pertinente, la participación en proyectos sociales no excluyentes".

La formulación e implementación de una política educativa orientada a la RSU supone grandes esfuerzos en pos de un modelo más responsable. Esto conlleva un replanteamiento del plano académico, con implicaciones en la forma en que se enseña y se aprende, esto es ajustes en el currículo; además de un esfuerzo decidido para la conformación de "comunidades de aprendizaje mutuo", en las que se involucren no solo los miembros de la comunidad universitaria (administrativos, estudiantes y los docentes) sino también los diferentes "públicos" (egresados, competidores, comunidades locales, proveedores de servicios, el Estado, entre otros) que mantienen vínculos con la organización.

REVISTA ANUAL ACCIÓN Y REFLEXIÓN EDUCATIVA, N 45

enero, 2020

ISSN L 2664-3775 


\section{EL EJE DE FORMACIÓN EN RESPONSABILIDAD SOCIAL UNIVERSITARIA}

La RSU debe converger en la formación integral de los estudiantes, preparándoles para entender las teorías que describen y explican los fenómenos de la realidad, pero también para fortalecer y consolidar la capacidad de identificar y resolver problemas morales en sus propias vidas, formar competencias para comprometerse e implicarse, escuchar y dialogar, saber mirar a través de los ojos del otro, aprender a ponerse en su lugar; pensar de forma crítica y empática, así como reflexionar (Vallaeys, 2012).

La RSU implica orientar la formación del estudiante hacia el desarrollo de su propia conciencia como un elemento importante en la solución de los problemas sociales, mediante acciones concretas que tomen en cuenta también al otro y su realidad; proporcionando competencias interpersonales para comprender a los demás, sentirse afectado y comprometido con las necesidades de su entorno (Vallaeys, De la Cruz y Sasia, 2009).

La universidad debe permitir que los individuos logren desarrollarse integralmente con el fin de que sus acciones contribuyan al mejoramiento de la sociedad. La formación profesional y humanística debe fomentar competencias de responsabilidad en sus egresados. Esto implica que la orientación curricular tenga una relación estrecha con los problemas reales (económicos, sociales, ecológicos) de la sociedad y esté en contacto con actores externos involucrados con dichos problemas. Ser socialmente responsable es comprometerse con el contexto (país, comunidad el mundo), intervenir y procurar mejorar las condiciones de vida y de convivencia.

Aún eso, el modelo educativo privativo de la sociedad de consumo en la que nos vemos inmersos, responde cada vez más a promover la competición y el individualismo. Es necesario un cambio hacia un esquema más crítico, basado en el entendimiento de las metas colectivas, el rescate de lo humano como prioritario y la mirada holística de los problemas.

REVISTA ANUAL ACCIÓN Y REFLEXIÓN EDUCATIVA, N 45

enero, 2020

SSN L 2664-3775 
Política de formación académica con enfoque de RSU para la

Universidad de Panamá, planificación para la acción.

En la declaración de la Conferencia Regional de Educación Superior en ALC (OEI, 2008) se señala que "Reivindicamos el carácter humanista de la Educación Superior, en función de la cual ella debe estar orientada a la formación integral de las personas, ciudadanos y profesionales, capaces de abordar con responsabilidad ética, social y ambiental los múltiple retos implicados en el desarrollo endógeno y la integración de nuestros países, y participar activa, crítica y constructivamente en la sociedad (IESALC- UNESCO, 2008).

La Responsabilidad Social Universitaria implica formar en los alumnos la capacidad de comprometerse, de escuchar y de diálogo, de saber mirar a través de los ojos del otro, de aprender a ponerse en su lugar, de tener un pensamiento crítico, capaz de identificar las partes de un todo y su interdependencia, de tener empatía, de entender el sentido auténtico del servicio, de la solidaridad y de la compasión, de "padecer con el otro" (De La Calle Maldonado; García Ramos; y Giménez Armentia, 2007).

Esto supone que los estudiantes se sometan a experiencias, del tipo teórico y práctico, que le permitan sensibilizarse con las personas y las condiciones socio-ambientales y culturales en las que conviven. Lo cual requiere el desarrollo de competencias en el plano de la comunicación, la cooperación, el trabajo en equipo, el liderazgo, la asertividad, la resolución de conflictos, la responsabilidad, el compromiso, la empatía, la organización, el uso responsable de los recursos y el autocontrol entre otras. Se trata de una formación basada en un curriculum sensible, que debe considerar el entorno, así como el autoaprendizaje y el aprendizaje colectivo, reflexivo y propositivo.

REVISTA ANUAL ACCIÓN Y REFLEXIÓN EDUCATIVA, N 45

enero, 2020

ISSN L 2664-3775 
La Responsabilidad Social Universitaria, implica como argumenta Ayala (2011, p. 33):

...una visión holística que articule las diversas partes de la institución en un proyecto de promoción social, de principios éticos y de desarrollo social equitativo y sostenible para la producción y transmisión de saberes para la formación de ciudadanos profesionales responsables.

Con ello la Universidad debe cumplir la misión de integrar un modelo formativo que promueva en su metodología de enseñanza/aprendizaje las situaciones de la realidad social que posibiliten el mejoramiento de las condiciones de vida de su sociedad, de su comunidad, y de su territorio.

A la Universidad, le corresponde "la función de conservar los valores permanentes del hombre, siendo una de sus funciones la de conjugar el progreso en los aspectos materiales de la vida con la defensa de los valores humanos del espíritu” (García Ramos, 1991). Según este autor, debe entenderse que: "la educación, depende de la concepción que se tenga del hombre y de su destino”. Sólo cuando se ha comprendido bien quién es el hombre en sí mismo y cuál es su meta última, se puede situar adecuadamente el problema de cómo guiarlo a la conquista de su meta personal. En este sentido se advierte sobre los aspectos que deben operativizarse para lograr la formación integral:

- en lo técnico, con la formación de profesionales altamente cualificados;

- en la investigación, con la búsqueda de nuevos conocimientos;

- en lo humano, con la defensa de valores fundamentales como la dignidad de la persona, el carácter sagrado de la vida, la libertad de pensamiento, de palabra y de profesar las propias convicciones, el papel central de la familia, la cooperación de todos para lograr el bien común, el valor del trabajo humano, la autoridad del Estado, gobernado por la ley y la razón;

REVISTA ANUAL ACCIÓN Y REFLEXIÓN EDUCATIVA, N 45

enero, 2020

SSN L 2664-3775 
Política de formación académica con enfoque de RSU para la

Universidad de Panamá, planificación para la acción.

- y en la formación de la conciencia y del sentido ético, integrándola con la formación técnica e intelectual.

La formación integral promueve una síntesis de saberes. Se trata de aprender a hacer para poder influir sobre el propio entorno, aprender a trabajar en equipo, para participar y cooperar con los demás en el cambio social, aprender a desarrollar plenamente sus propias capacidades con sentido de responsabilidad, para que tengan una participación en la sociedad. Formar personas que además de ser capaces de reconocerse (talento, intereses, valores, aspiraciones y debilidades), se muestren comprometidas con su entorno y con sus semejantes.

En la formación integral se destaca "la formación humanista centrada en el desarrollo profesional como ser humano que se construye en el ejercicio de una profesión y como tal se desempeña en ella” (Hernández Fernández, 2013, p. 24).

La responsabilidad social es una abstracción, un valor, por tanto, una persona no es socialmente responsable si sólo conoce intelectualmente el concepto; la responsabilidad social se ejerce y concreta a través de una conducta moral (Navarro, 2002). Aristóteles, apunta a sostener que una persona no es moral si únicamente conoce intelectualmente el bien; para serlo es preciso que mantenga una línea de conducta virtuosa: que realice actos virtuosos y que los realice habitualmente, es decir, actos que apunten en dirección al bien, a la supervivencia y a la felicidad del hombre y de la especie humana. Estos actos virtuosos son también llamados conductas constructivas y son aquellas que contribuyen a satisfacer las propias necesidades de autoprotección y desarrollo y, a la vez, cooperar en la satisfacción de las necesidades de los demás, para una plena y sana supervivencia (Navarro, 2003).

La universidad debe estar centrada en dotar de conocimiento a sus alumnos con el fin de su realización personal, de forma ética, y socialmente responsable hacia su desempeño profesional. En este sentido el estudiante debe alcanzar cierta madurez intelectual como emocional que le

REVISTA ANUAL ACCIÓN Y REFLEXIÓN EDUCATIVA, $\mathrm{N}^{\circ} 45$

enero, 2020

ISSN L 2664-3775 
permita conocer y reconocer el impacto de sus acciones personales y profesionales, así como reconocer y hacerse responsable de las consecuencias de su actuar.

\section{Aspectos metodológicos}

Tradicionalmente, las políticas de educación son estudiadas bajo la rigurosidad de metodologías cuantitativas, recurriendo al uso de instrumentos estadísticos. Sin embargo, cuando hablamos de resolver y analizar problemas referidos a la calidad de la educación, es posible incluir un enfoque cualitativo. La investigación que se propone en este sentido es del tipo descriptiva ya que trabaja sobre realidades de hechos, procurando una interpretación y/o caracterización indicando sus rasgos más peculiares o diferenciadores.

Para este estudio se procuró la aplicación de instrumentos de investigación cualitativos basados en: entrevistas semiestructurada dirigida a líderes del proyecto, encuestas de 10 preguntas dirigida a docentes (modelo de encuesta adaptado de Valleays, de la Cruz y Sasia; 2009), y el análisis de documentos.

Los documentos que sirven de referencia para esta investigación son aquellos que definen los conceptos generales, impactos, actores, ejes de RSU entre otros y algunos relacionados con la implementación de proyectos en la región latinoamericana, investigaciones realizadas, así como aquellos relacionados con los temas de políticas educativas. De igual forma servirán de análisis documentos institucionales de la UP: Textos, Boletines informativos, Revistas, Plan de Desarrollo Institucional de la Universidad de Panamá, Estatuto Universitario, Plan de Mejoramiento Institucional, Informe de Autoevaluación 2012, Informes de la Vicerrectoría Académica, Modelo Académico Curricular.

REVISTA ANUAL ACCIÓN Y REFLEXIÓN EDUCATIVA, N 45

enero, 2020

SSN L 2664-3775 
Política de formación académica con enfoque de RSU para la

Universidad de Panamá, planificación para la acción.

\section{Muestreo por conveniencia}

El muestreo aleatorio, fue aplicado a docentes por conveniencia, en cuanto a que facilitó la aplicación de la técnica de la encuesta, volcándose en ella, las valoraciones asumidas y emitidas por 67 docentes que laboran en 17 facultades en ambos Campus Universitarios de la Universidad de Panamá (Octavio Méndez Pereira y Harmodio Arias Madrid). Dicha población objeto de estudio es aquella relacionada directamente con la implementación del proyecto de transformación curricular.

\section{ANÁLISIS DE RESULTADOS}

\section{Entrevistas}

La entrevista semi estructurada fue aplicada a: la Coordinadora del Proyecto de Transformación Curricular 2004-2016, y la jefa del Programa; quienes refiriéndose al alcance del proyecto y su relación con la RSU señalaron que:

- En el año 2004 se inicia el proceso de transformación académica curricular (Consejo Académico en la Sesión № 44-04 del 27 de octubre de 2004), que con la aprobación de la Política Académica 2007-2011 se formaliza y cobra fuerza.

- El Modelo Educativo y Académico promulgado en 2006 (que fue ratificado mediante Acuerdo $\mathrm{N}^{\circ} 32$ del 17-8-2011), sentó las bases y brindó directrices para el trabajo, que por 16 años se ha realizado de manera continua, en las distintas Facultades, Centros Regionales y Extensiones Docentes.

REVISTA ANUAL ACCIÓN Y REFLEXIÓN EDUCATIVA, N 45

enero, 2020

ISSN L 2664-3775 
- Se destaca también que "el proceso de transformación del curriculum implementado, es la primera acción que de forma sistemática e integral busca mejorar la calidad de la oferta académica de la Universidad de Panamá".

- Las orientaciones teóricas y metodológicas que sustentan las acciones se fundamentan en los lineamientos y disposiciones legales y normativas de la Ley Orgánica y el Estatuto Universitario, así como en enfoques y aportes desde diversas disciplinas acerca de las exigencias de la educación superior para el siglo XXI.

- Desde esas perspectivas, la Política y el Proyecto de Transformación Académica y Curricular tiene claramente definido que la formación de los profesionales debe asegurar:

...profesionales íntegros, generadores de conocimientos con alto compromiso social y creadores de iniciativas que coadyuven a mejorar el bienestar y calidad de vida de los panameños. Es decir, formar profesionales con las competencias sociales de convivencia ciudadana; comprometidos con la protección, uso racional y sostenible del medio ambiente; con valores socialmente aceptados; integrados solidariamente al desarrollo socioeconómico y cultural de país; con una personalidad equilibrada en los aspectos éticos, intelectuales y socio afectivos de la cultura humana. (Universidad de Panamá, 2017).

- Para concretar tales finalidades en la Política de Transformación del Curriculum se ha establecido que en cada oferta de formación existen rasgos de perfil comunes que distinguen al egresado de la Universidad de Panamá, los que aparecen a continuación:

- Demostrar plena conciencia de su condición personal y profesional para el cumplimiento cabal de su proyecto de vida desde su particular esfera de

REVISTA ANUAL ACCIÓN Y REFLEXIÓN EDUCATIVA, N 45

enero, 2020

SSN L 2664-3775 
Política de formación académica con enfoque de RSU para la

Universidad de Panamá, planificación para la acción.

actuación, cimentado en una conciencia ética y valores morales en aras de la construcción de una sociedad más justa, equitativa y solidaria.

- Manifestar actitudes, valores e ideales ciudadanos de vida democrática contribuyendo con su participación social comprometida con las tareas de consolidación del estado nacional independiente, con el desarrollo sostenible, la equidad y la justicia social en el marco de la cultura de paz (Universidad de Panamá, 2004)

- Uno de los aspectos en los que se ha trabajado es en la redefinición de los perfiles de formación a fin de adecuarlos a las nuevas tendencias y demandas sociales mediante el enfoque de formación por competencias. En dicha tarea se establecen los conocimientos, habilidades, actitudes, valores que debe poseer todo profesional egresado de esta casa de estudios. Para ello se incluyen competencias básicas, genéricas y específicas. Entre las competencias genéricas se encuentran algunas que refieren a RSU, tales como:

Competencias Genéricas que son un referente regional para la formación de profesionales de todas las carreras.

13. Capacidad para actuar en nuevas situaciones.

14. Capacidad creativa.

20. Compromiso con la preservación del medio ambiente.

21. Compromiso con su medio socio-cultural.

22. Valoración y respeto por la diversidad y multiculturalidad.

26. Compromiso ético" (Proyecto Tuning América Latina).

- Estrategia de transversalidad en el curriculum desde la cual se considera la inclusión a través de las distintas asignaturas el fomento de valores, ideales, actitudes de manera

REVISTA ANUAL ACCIÓN Y REFLEXIÓN EDUCATIVA, N 45

enero, 2020

ISSN L 2664-3775 
holística y permanente. Los temas transversales son contenidos a lo largo de la toda la escolaridad que deben permitir que el egresado posea un pensamiento interdisciplinar y transdisciplinar de las temáticas y problemas que afectan la vida en sociedad y en especial, a los sectores más desposeídos, en correspondencia con el compromiso histórico establecido desde su fundación para esta casa de estudios. Los ejes articuladores de la transversalidad directamente vinculados a RSU son los siguientes:

-Educación para la paz y la comprensión intercultural;

-Educación ambiental;

-Educación ciudadana y patriótica;

-Educación para la salud y

-Educación para el trabajo productivo y creador.

- Núcleo Común de Formación: Como parte de las transformaciones en los planes y programas de estudio donde está también claramente establecido el enfoque de RSU se puede mencionar la aprobación por el Consejo Académico de un Núcleo Común de Formación que entre sus finalidades se dirige a formar en todas las carrearas asignaturas dirigidas al logro de competencias ciudadanas de RSU; aparecen como obligatorias las asignaturas de Medio Ambiente y Desarrollo Sostenible, Historia y Geografía de Panamá en las cuales se estudian los principales problemas sociales y ecológicos y se presentan las alternativas de solución.

- Según la Vicerrectoría Académica, como producto de la Transformación Académica Curricular (TAC) se han creado 56 nuevas carreras y se han actualizado 65. Ello representa el $67.97 \%$ en ambos procesos. E1 32,02\% de la oferta restante está en distintos niveles de avance en el proceso de actualización (Universidad de Panamá, 2016).

REVISTA ANUAL ACCIÓN Y REFLEXIÓN EDUCATIVA, N 45 enero, 2020 
Política de formación académica con enfoque de RSU para la

Universidad de Panamá, planificación para la acción.

Así mismo señala la Vicerrectoría Académica que:

Según la naturaleza de la carrera y/ asignatura, el tipo de trabajo que realizan profesores y alumnos durante el proceso formativo, pudiera decirse que en algunas de ellas desde hace tiempo se realizan actividades pedagógicas que se enmarcan en este enfoque de RSU, aun cuando no se contemple en la práctica bajo tal denominación. En estos casos puede hacerse de manera sistemática en unas y ocasionalmente en otras dentro de una carrera $o$ asignatura.

Por otro lado, hay carreras en donde no se cumple en lo absoluto con la proyección social desde esta perspectiva o ninguna. Entre estos extremos de seguro habrá una variedad de posibilidades.

Se recomienda por tanto que:

El tema de responsabilidad social universitaria deberá ser motivo de mayor conocimiento y aplicación en lo que al currículum se refiere, lo que implicaría entre otros asuntos de importancia, la capacitación de los docentes y de quienes gestionan en currículum a todos los niveles a fin de que la temática se vaya colocando gradualmente en la cultura universitaria.

Finalmente, respecto a la identificación de experiencias de incorporación del enfoque de RSU desarrolladas durante el proceso de Transformación Curricular que puedan facilitar lineamiento para política de RSU en cuanto a formación académica; la encargada de la divulgación del proyecto nos refiere a "Estrategia"- Boletín Informativo de Transformación Curricular, que en sus 87 ediciones evidencia un conjunto de actividades que bien pudieran insertarse como de $\mathrm{RSU}$. Al respecto nos comenta que un rápido recorrido a algunos de los ejemplares (disponibles

REVISTA ANUAL ACCIÓN Y REFLEXIÓN EDUCATIVA, N 45

enero, 2020

ISSN L 2664-3775 
en la web institucional dentro de link de Vicerrectoría Académica) evidencia que las experiencias de RSU se muestran en términos de: Giras de trabajo de campo a comunidades vulnerables, exposiciones, conferencias, investigaciones, pasantías; atendiendo temas relativos a salud (control de vectores, VIH- SIDA), salud animal, ambiente (reforestación, cambio climático), desarrollo comunitario, cultura de paz entre otros.

\section{Encuestas}

Entre 2016-2017, se aplicaron un total de 67 encuestas al azar, a docentes de 17 de 19 Facultades de la Universidad de Panamá (Medicina Veterinaria y Farmacia no participaron en la muestra). Se trata de profesores de licenciaturas y otros de nivel de postgrado. El instrumento utilizado para realizar las encuestas tomó como referencia el Manual de Primeros Pasos (Valleays, De la Cruz y Sasia, 2009) para la autoevaluación de la RSU, con ajustes.

La Figura 1, muestra la representatividad de los profesores, según 17 facultades de la Universidad de Panamá. 
Política de formación académica con enfoque de RSU para la Universidad de Panamá, planificación para la acción.

\section{Figura 1}

Profesores encuestados por facultad

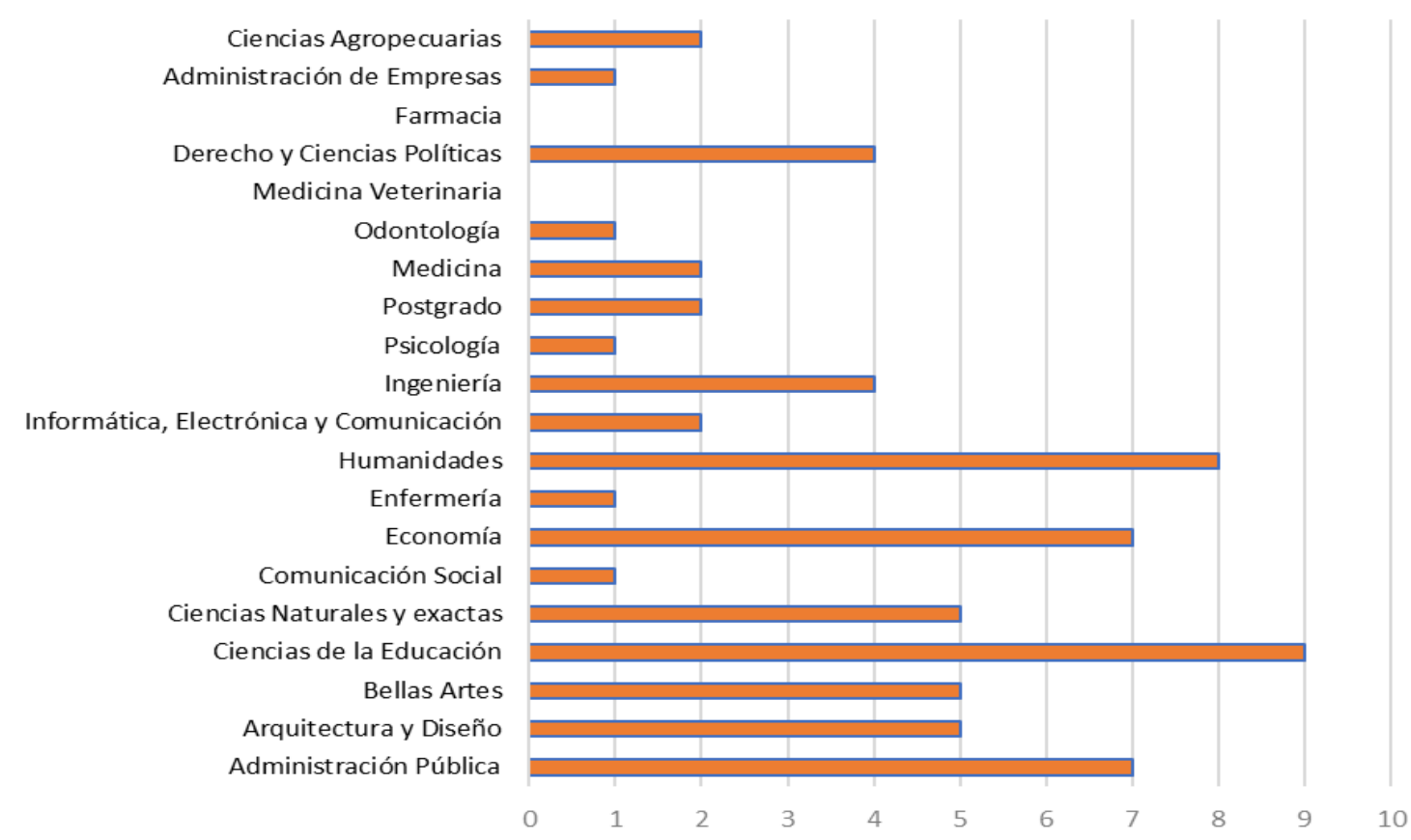

Fuente: información obtenida de encuesta aplicada. 2016.

Frente a la consulta de si la Universidad brinda una formación ética y ciudadana, el $88 \%$ de los entrevistados dice estar total o parcialmente de acuerdo. Esta condición podría suponer, que el componente ético y ciudadano en la formación está presente como parte de los objetivos y la estrategia de aprendizaje, en términos de competencias para la formación profesional.

REVISTA ANUAL ACCIÓN Y REFLEXIÓN EDUCATIVA, N 45

enero, 2020

ISSN L 2664-3775 


\section{Figura 2}

Formación ética de los estudiantes

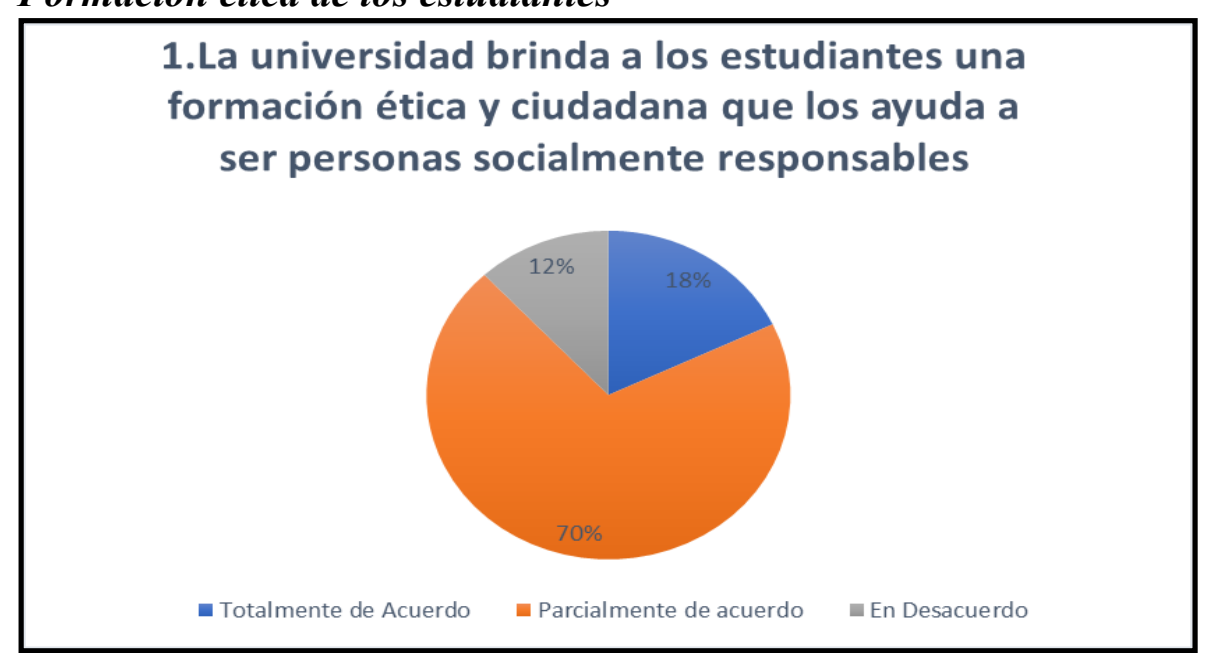

Fuente: información obtenida de encuesta aplicada. 2016.

Aún lo anterior, la respuesta a la pregunta 3, evidencia que la gran mayoría de los profesores (un $55 \%$ ), percibe que de forma "parcial" los estudiantes parecen estar informados de las injusticias sociales y los riesgos ecológicos actuales.

\section{Figura 3}

Percepción acerca de información que manejan los estudiantes en materia social y ambiental

\section{Percibo que los estudiantes están bien informados acerca de las injusticias sociales y los riesgos ecológicos del mundo actual.}

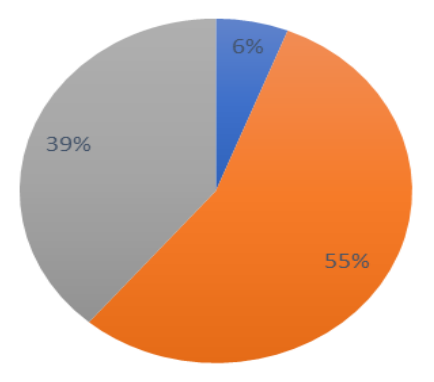

- Totalmente de Acuerdo

- Parcialmente de acuerdo

En Desacuerdo

Fuente: información obtenida de encuesta aplicada. 2016.

REVISTA ANUAL ACCIÓN Y REFLEXIÓN EDUCATIVA, N 45 enero, 2020 
Política de formación académica con enfoque de RSU para la

Universidad de Panamá, planificación para la acción.

Un aspecto importante en la formación en RSU es la coordinación entre colegas y por departamentos, para el desarrollo de estrategias de enseñanza que aseguren el aprendizaje integral y reflexivo de la realidad social vinculada al campo profesional. La respuesta a la pregunta 2, evidencia problemas de coordinación entre pares que podrían ameritar acciones en materia de capacitación que aseguren una mejor gestión de lo curricular a partir del trabajo colaborativo para la articulación de la responsabilidad social.

\section{Figura 4}

Reuniones con colegas para examinar RSU

2. He tenido reuniones con colegas para examinar los aspectos de responsabilidad social ligados a la carrera que enseño.

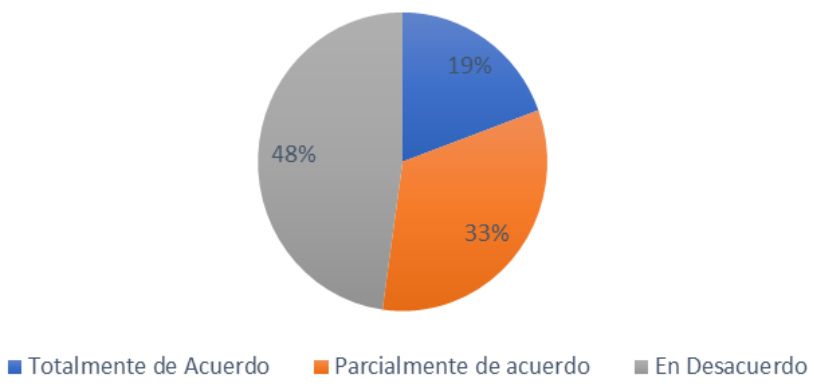

Fuente: información obtenida de encuesta aplicada. 2016.

La consulta acerca de la incorporación de acciones vinculadas con la RSU a lo interno de los cursos evidencia que buena parte de los profesores indican que han actualizado sus cursos atendiendo a la temática social (50\%), algunos lo han hecho parcialmente y solo $12 \%$ señala que no lo ha realizado.

Esto parece corresponderse con el hecho de que los contenidos temáticos, se señala, atienden lo social y ambiental (70\%), aunque aún, las actividades planificadas tengan poco impacto con el entorno (43\%) y solo un $27 \%$ de los cursos no reporten vínculos con proyectos sociales fuera de la universidad. 
Lo anterior evidencia que el Proyecto de Transformación Curricular que condujo a la revisión y actualización de los programas y carreras ha obligado a replantear los cursos hacia objetivos más vinculantes con el entorno social.

\section{Figura 5}

Desarrollo de acciones de RSU a lo interno de los cursos

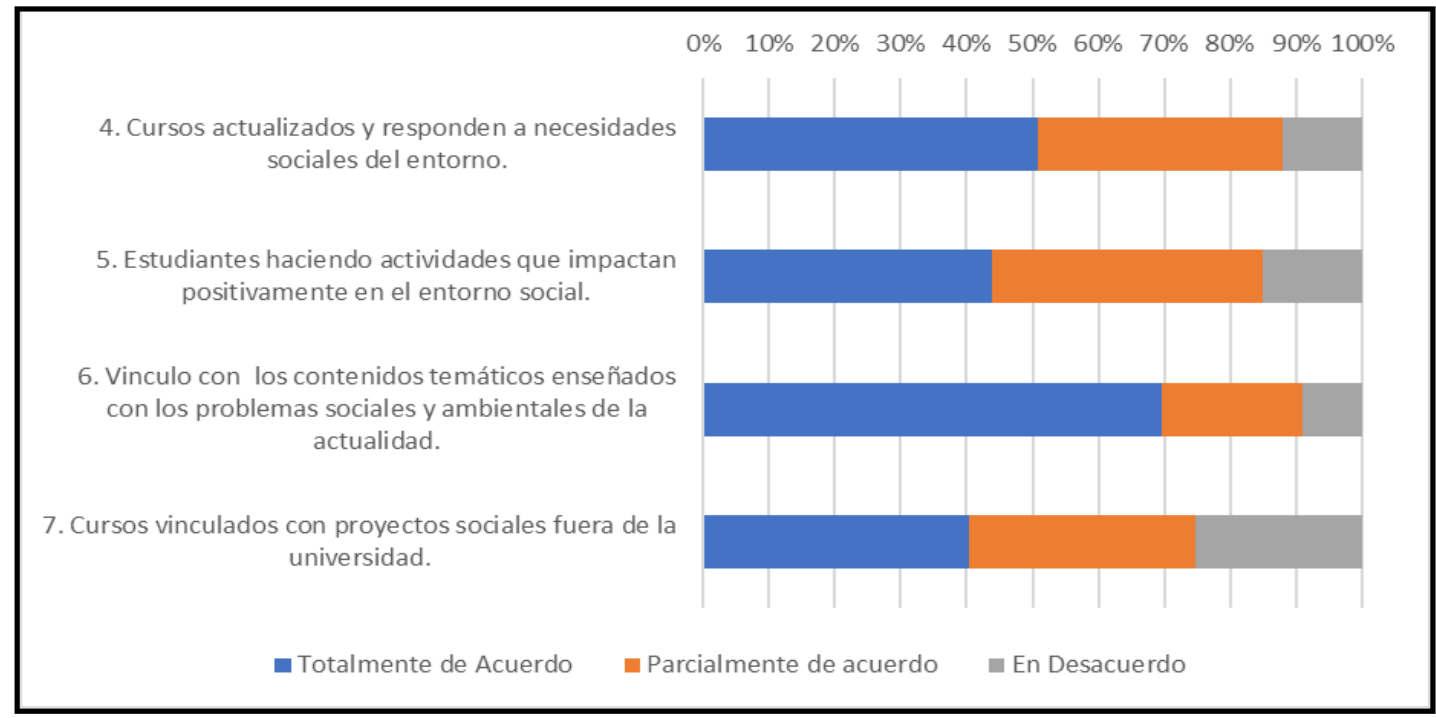

Fuente: información obtenida de encuesta aplicada. 2016.

En cuanto al establecimiento de vínculos con actores sociales a lo externo de la universidad un $40 \%$ señala estar en desacuerdo en que se hace, sin embargo, cuando se le indaga acerca de los vínculos con los egresados para temas de adecuación del currículum, es mayor el porcentaje que indica que no se logra (52\% señala que no se realiza).

REVISTA ANUAL ACCIÓN Y REFLEXIÓN EDUCATIVA, N 45 enero, 2020 
Política de formación académica con enfoque de RSU para la

Universidad de Panamá, planificación para la acción.

\section{Figura 6}

Vinculos con actores externos y egresados

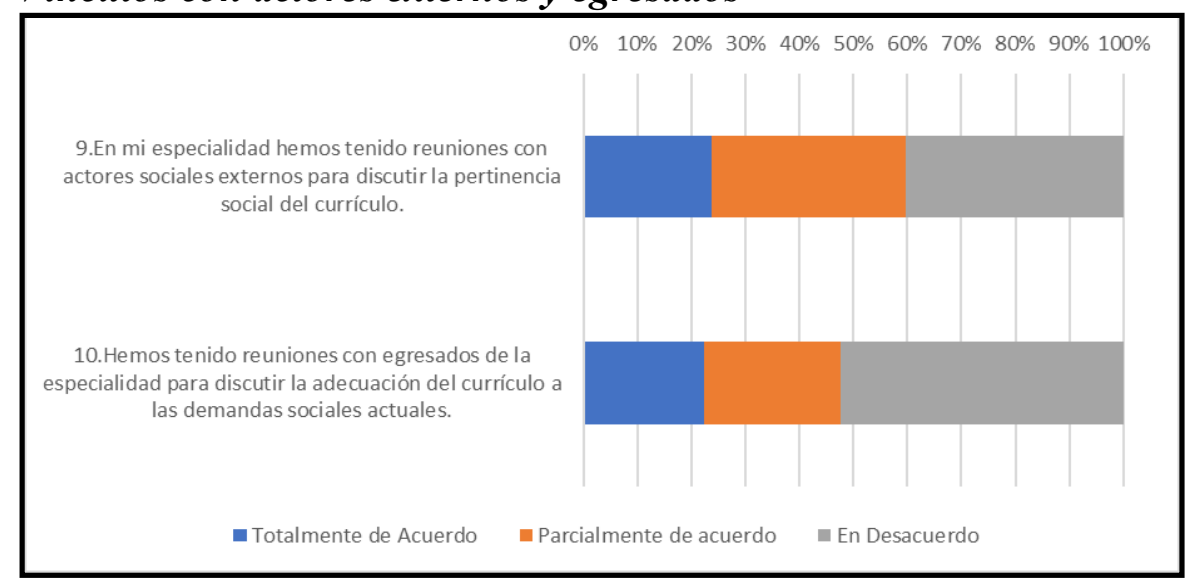

Fuente: información obtenida de encuesta aplicada. 2016.

Finalmente, y respecto al voluntariado se evidencia que, aunque parece realizarse no está del todo articulado a las actividades curriculares.

\section{Figura 7}

Participación en actividades de voluntariado solidario

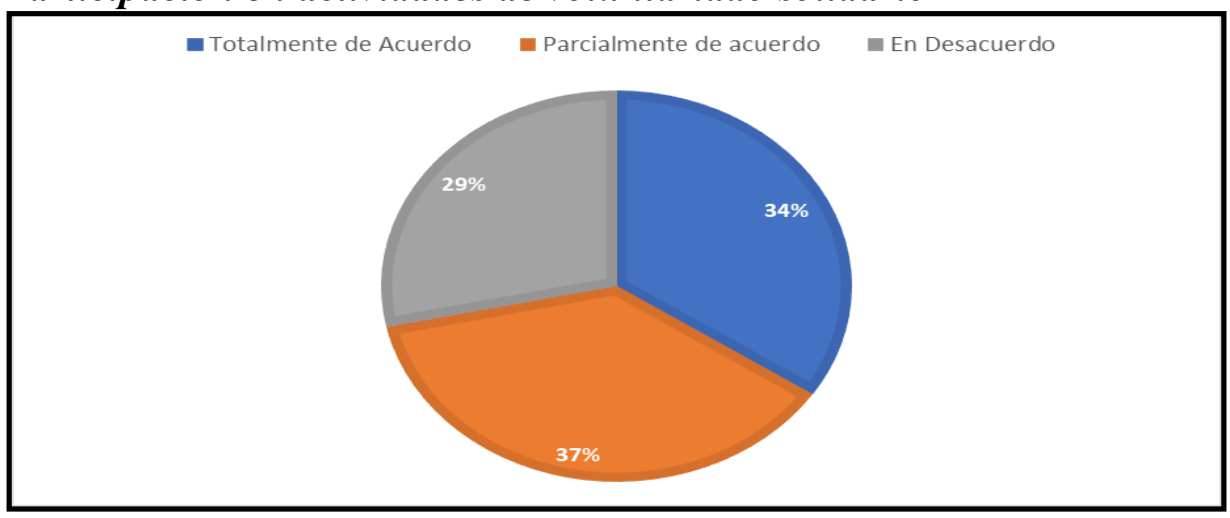

Fuente: información obtenida de encuesta aplicada. 2016. 


\section{Análisis Documental}

Para el análisis documental se recurrió a la revisión de documentos de: política universitaria, en este caso, la Política Universitaria 2007-2012, en especial lo relativo a la Política de Responsabilidad Social Universitaria (2007-2011, 2012-2016 y 2017-2020); normas, tales como: Ley 24 de 14 de julio de 2005- Universidad de Panamá, el Estatuto Universitario (aprobado por el Consejo General Universitario el 29 de octubre de 2008), el Reglamento de Servicio Social (aprobado por Consejo Académico Reunión 41-07 del 1 de agosto de 2007), así como el Modelo Educativo y Académico de la Universidad de Panamá- 2008 y el Informe Final de Acreditación de la Universidad de Panamá de 2012.

Todos estos documentos se evaluaron en función de 4 aspectos distintivos de la RSU para lo académico:

- Presencia de temáticas ciudadanas y de responsabilidad social en el currículo (DD.HH., desarrollo sostenible, ética profesional y cívica, gestión de la RS, etcétera).

- Articulación entre profesionalización y voluntariado solidario.

- Aprendizaje profesional basado en proyectos sociales.

- Integración de actores sociales externos en el diseño de las mallas curriculares.

Es importante resaltar que en casi todos los documentos se observó vinculación con los cuatro aspectos analizados, destacándose con ello, no solo la presencia de las temáticas de RSU, sino la propuesta curricular dirigida hacia aprendizajes basados en proyectos y la permanente vinculación con actores sociales para la definición de las mallas curriculares.

Aún eso los aspectos de política de RSU desde el 2008 y los propuesto hasta el 2020, inclinan el tema de la responsabilidad social hacia la extensión, desvinculándola de lo académico curricular. 
Política de formación académica con enfoque de RSU para la

Universidad de Panamá, planificación para la acción.

En lo relativo a la "articulación entre la profesionalización y el voluntariado" poco se evidencia. El concepto de voluntariado como acción de la universidad solo se menciona en el Informe Final de Acreditación. Esto quizás por la preminencia del Servicio Social, legalizado desde 2007, que se presenta como una acción más contundente de relación universidad - sociedad. El Servicio Social es realizado por los estudiantes, como parte de su pensum, sin recibir remuneración económica o prestaciones laborales y se ejecuta en beneficio de las comunidades y otros sectores que lo requieran, privilegiando a las clases más necesitadas.

\section{Discusión}

El Proyecto de Transformación Curricular iniciado en 2004, conduce y orienta la Política universitaria de la Universidad de Panamá para el período 2007-2011 en lo académico curricular, y logra su articulación con lo propuesto en términos de Política de Responsabilidad Social Universitaria. Esto se evidencia en la propuesta del Proyecto de Fortaleciendo los Planes y Programas de Estudio desde el enfoque de la Responsabilidad Social Universitaria, para el 20072011.

Se trata de la primera acción, que de forma sistemática e integral busca mejorar la calidad de la oferta académica de la Universidad de Panamá procurando el logro de la misión y visión universitarias. La misma se basa en orientaciones teóricas y metodológicas que se fundamentan en los lineamientos y disposiciones legales y las normativas universitarias, así como en las exigencias de calidad para la educación superior para el siglo XXI (incluyen la responsabilidad social universitaria).

El proyecto considera cambios profundos en lo curricular tendiente a incorporar la RSU a partir del desarrollo de habilidades sociales, expresadas en la relación con la sociedad, la educación para la conciencia social y el entendimiento de lo global y la exploración del mundo real, asegurando oportunidades de contribución y compromiso social. Esto queda claramente

REVISTA ANUAL ACCIÓN Y REFLEXIÓN EDUCATIVA, $\mathrm{N}^{\circ} 45$

enero, 2020

ISSN L 2664-3775 
evidenciado en: el Enfoque de formación por competencias, que incluye algunas referidas directamente con la RSU, la consecución de un Perfil Común que diferencie al egresado de la Universidad de Panamá en términos de "manifestar actitudes, valores e ideales ciudadanos de vida democrática contribuyendo con su participación social comprometida con las tareas de consolidación del estado nacional independiente, con el desarrollo sostenible, la equidad y la justicia social en el marco de la cultura de paz"; en el desarrollo de una Estrategia de Transversalidad en el Curriculum tendiente a promover un pensamiento interdisciplinar y transdisciplinar de las temáticas y problemas que afectan la vida en sociedad y en especial, a los sectores más desposeídos, en correspondencia con el compromiso histórico de la Universidad de Panamá; en la propuesta de inserción del Núcleo Común de Formación que coloca como obligatorias las asignaturas de Medio Ambiente y Desarrollo Sostenible, Historia y Geografía de Panamá en las cuales se estudian los principales problemas sociales y ecológicos buscando de esta forma articular el pensamiento crítico hacia la solución de problemas de la comunidad panameña.

Es claro que el Proyecto de Trasformación Curricular procura la integralidad en la formación de profesionales, con fortalezas en lo humanístico, así como en lo técnico científico; para lo cual considera el vínculo estrecho con la investigación y la extensión, con el fin de procurar conocimiento útil, con alto compromiso social y ético. Profesionales creadores de iniciativas que coadyuven a mejorar el bienestar y calidad de vida de los panameños, atendiendo con ello a la misión y visión de la Universidad de Panamá.

Durante el análisis documental pudo evidenciarse la incorporación del enfoque de RSU en los documentos de política y normativa universitaria, lo cual podría sustentar la formulación y desarrollo de acciones subsecuentes, encaminadas a dar forma a una política más robusta en materia curricular con enfoque de RSU.

REVISTA ANUAL ACCIÓN Y REFLEXIÓN EDUCATIVA, $\mathrm{N}^{\circ} 45$

enero, 2020

SSN L 2664-3775 
Política de formación académica con enfoque de RSU para la

Universidad de Panamá, planificación para la acción.

Del análisis de las encuestas a los profesores de las diferentes facultades aplicadas en 2016, se observa el compromiso de los docentes por vincular aspectos de responsabilidad social en sus cursos, tanto a nivel de la pertinencia y actualización de los contenidos temáticos, así como en las estrategias didácticas orientadas a los contextos.

Un hallazgo interesante es la poca inserción del componente de voluntariado en la vida universitaria, esto quizás debido a la formalización del Servicio Social obligatorio. El Servicio Social obligatorio (vigente en UP desde el 2007) es una oportunidad para la inserción de la RSU, en su estructuración y fines permite una plataforma eficiente para orientar procesos de formación profesional responsable. Aún eso, por ocurrir al final de la carrera, no puede ser considerado como único. Y debe ser antecedido y complementado con otras acciones que, a lo largo de la formación, permitan un mejor desarrollo de propuestas para su ejecución.

Por otro lado coincidimos, en que según la naturaleza de la carrera y/ asignatura, el tipo de trabajo que realizan profesores y alumnos, en la actualidad, durante el proceso formativo, en algunos casos y desde hace tiempo, consideran el desarrollo de actividades pedagógicas que se enmarcan en el enfoque de RSU, de esto da muestra las 87 ediciones del Boletín Informativo de Transformación Curricular; en donde se evidencia el desarrollo de actividades que bien pudieran insertarse como de RSU tales como: Giras de trabajo de campo a comunidades vulnerables, exposiciones, conferencias, investigaciones, pasantías; atendiendo temas relativos a salud (control de vectores, VIH- SIDA), salud animal, ambiente (reforestación, cambio climático), desarrollo comunitario, cultura de paz entre otros.

Aún lo anterior es necesario dar mayor coherencia a las estrategias didácticas implementadas, por lo cual urge promover el fortalecimiento de las capacidades de los docentes en el uso de metodologías participativas que aseguren el aprendizaje colaborativo, dirigido a la resolución de problemas reales /complejos del contexto socioeconómico de las poblaciones y los ecosistemas,

REVISTA ANUAL ACCIÓN Y REFLEXIÓN EDUCATIVA, N 45

enero, 2020

ISSN L 2664-3775 
procurando además la orientación al servicio; esto con el fin de asegurar el carácter activo y propositivo del estudiante y de la educación basada en valores. En esta misma línea es necesario ahondar aún más en la concreción del perfil del docente para la implementación del RSU, fortaleciendo capacidades de los profesores para tomar decisiones a partir de la reflexión acerca del contexto y las características del curso.

\section{Conclusión}

Preocupa, que aún los avances en materia de RSU y transformación curricular articulados y propuestos para la política universitaria 2007-2011; en los sucesivos dos planes estratégicos de la Universidad de Panamá 2012-2016 y 2017-2021 aunque se declara el componente de RSU, este se observa más que nada vinculado a acciones de divulgación de la "cultura de RSU" y como una acción prioritariamente de Extensión, dejándose de lado el "eje de formación".

Los docentes muestran inquietud por establecer permanentes y más estables vínculos a lo externo de la universidad (sociedad y egresados), que permitan la pertinencia social de los diseños curriculares de las carreras y en consecuencia los programas de las asignaturas; este aspecto merece resaltarse, dado el rol de importancia, que este actor tiene, para el logro de la implementación de la política.

Hace falta mayor estímulo a la comunicación y el establecimiento de espacios para el trabajo interdisciplinario a lo interno y externo de las carreras que faciliten la inserción de la RSU de forma óptima. Esto supone además la movilización de lo administrativo para subsanar estas debilidades.

Finalmente, mientras no se entienda la relevancia y complejidad de la RSU en el quehacer universitario poco podrá avanzarse para el diseño de un modelo a tono con el carácter de nuestra

REVISTA ANUAL ACCIÓN Y REFLEXIÓN EDUCATIVA, $\mathrm{N}^{\circ} 45$

enero, 2020

SSN L 2664-3775 
Política de formación académica con enfoque de RSU para la

Universidad de Panamá, planificación para la acción.

institución (principal universidad pública del país), aun cuando de forma declarativa los documentos de política lo señalen. Por ello hace falta una mayor sensibilización de las autoridades y de la comunidad educativa.

Para el diseño e implementación de una política académica curricular con enfoque de RSU en la Universidad de Panamá, es perfectamente rescatable y necesario continuar los procesos de Transformación curricular, siempre y cuando se vaya de la mano acciones tendientes al fortalecimiento de los equipos de comunicación y gestión curricular, la capacitación de los docentes y la inserción de los alumnos y personal administrativo. Aspectos estos esenciales para el éxito de una acción de política.

\section{Referencias}

Ayala García, Mauricio Osvaldo. (2011). Responsabilidad social universitaria. El Salvador. Universidad Francisco Gavidia. Revista Realidad y Reflexión $N^{\circ}$ 33. Septiembre a diciembre. 2011.

De la Calle Maldonado de Guevara, C. y Otros. (2007). "La formación de la responsabilidad social en la universidad". Revista Complutense de Educación. Vol. 18 Núm. 2 (2007), pp. 47-66.

Disponible

en https://www.researchgate.net/publication/27593658_La_formacion_de_la_responsabilidad_s ocial_en_la_universidad/.

García Ramos, J. (1991). "La formación integral: objetivo de la Universidad: Algunas reflexiones sobre la educación en la Universidad". Revista Complutense de Educación, m, Vol. 2 (2) - pp. 323-335.

REVISTA ANUAL ACCIÓN Y REFLEXIÓN EDUCATIVA, N 45

enero, 2020

ISSN L 2664-3775 
Hernández Fernández, H.; Hernández, A.; y González, M. (2013). La formación profesional en el contexto de la educación superior cubana. Características principales. En Aportes de Enfoque histórico-cultural a la educación. Experiencias de su aplicación en la Universidad de La Habana. Cuba: Editorial FEDUN.

Navarro, G. (2002). "Universidad Construye País: una experiencia de cooperación entre las Universidades Chilenas". Ponencia presentada al Seminario Nuevos Modelos de Cooperación Social: un enfoque socio jurídico. Evento realizado en Oñati-España, entre el 19 y 20 de septiembre.

Navarro, G. (2003). Educación para la responsabilidad social: Elementos para la discusión. Chile: Universidad de Concepción.

Organización de Estados Iberoamericanos -O.E.I.- (2008). Declaración Final de la Conferencia Regional de Educación Superior en América Latina y El Caribe Conferencia Regional de Educación Superior.

Tünnermann B., C. (2009). La Educación Superior necesaria para el Siglo XXI. Revista Temas No. 57, (Pp. 42-51).

UNESCO. (1998). Declaración Mundial sobre Educación Superior en el Siglo XXI: Visión y Acción. Conferencia Mundial sobre Educación Superior. Recuperado de www.unesco.org/education/educprog/wche/declaration_spa.htm

UNESCO. (2009). II Conferencia Mundial sobre la Educación Superior - 2009: La nueva dinámica de la educación superior y la investigación para el cambio social y el desarrollo. Recuperado de www.unesco.org.ve/dmdocuments/comunicado_cmes09es.pdf/.

REVISTA ANUAL ACCIÓN Y REFLEXIÓN EDUCATIVA, N 45 enero, 2020 
Política de formación académica con enfoque de RSU para la

Universidad de Panamá, planificación para la acción.

UNESCO-IESALC. (2007). Congreso Internacional de Rectores Latinoamericanos y Caribeños. Declaración Final de Bello Horizonte.

UNESCO-IESALC. (2008). Tendencias de la Educación Superior en América Latina y el Caribe. Bogotá: Panamericana. Formas e Impresos S.A.

Universidad de Panamá. (2018). Plan de Desarrollo Institucional 2017-2021.

Universidad de Panamá. (2016). Informe Final. La Transformación Académica Curricular 20042013. Plan de Desarrollo Institucional 2012-2016.

Universidad de Panamá. (2009). Modelo Educativo y Académico de la Universidad de Panamá. Ratificado mediante Acuerdo $\mathrm{N}^{\circ} 32$ del 17-8-2011.

Universidad de Panamá. (2009). Reglamento del Servicio Social de la Universidad de Panamá Aprobado por Consejo Académico Reunión 41-07 del 1 de agosto de 2007 y por el Consejo General Universitario N ${ }^{\circ}$ 2-10 del 2 de marzo de 2009.

Universidad de Panamá. (2008). Plan de Desarrollo Institucional 2007-2011.

Universidad de Panamá. (2008). Estatuto Universitario. Aprobado por el Consejo General Universitario el 29 de octubre de 2008).

Universidad de Panamá. Ley 24 de 14 de julio de 2005- Universidad de Panamá.

Universidad de Panamá. (2004). Política y Proyecto de Transformación Académica Curricular. Universidad de Panamá. Consejo académico, Sesión N 44-04 del 27 de octubre de 2004.

REVISTA ANUAL ACCIÓN Y REFLEXIÓN EDUCATIVA, N 45

enero, 2020

ISSN L 2664-3775 
Vallaeys, F.; De La Cruz, C.; y Sasia, P. (2009). Responsabilidad social universitaria: manual de primeros pasos. México: McGraw Hill. 